\title{
Effect of quark off-shellness in DIS and the Drell-Yan process
}

\author{
O. Linnyk, S. Leupold, U. Mosel \\ Institut für Theoretische Physik, Universität Giessen, Germany
}

August 3, 2018

\begin{abstract}
We study higher twist corrections to the perturbative QCD cross sections for $e p$ deep inelastic scattering and the Drell-Yan lepton pair production in $p p$ collision. The corrections arise due to the initial state interaction of the active quarks with the spectator partons. The effect of this interaction is calculated by dressing the incoming quark lines with phenomenological single parameter Breit-Wigner spectral functions and taking into account full off-shell kinematics. The quark width in the proton is estimated by analyzing the data on Drell-Yan triple differential cross section from the experiment E866 at Fermilab.
\end{abstract}

There is an analogy between the quasi-elastic e-nucleus scattering at high energy and the deep inelastic ep scattering(DIS). In the plane wave impulse approximation(PWIA) of nuclear physics, the electron is believed to interact with single nucleon in the nucleus, if the energy transfer is much higher than the binding energy. Inclusive $e A$ cross sections can be calculated from a handbag diagram, neglecting the interaction of the struck nucleon with the rest of the nucleus. In this approximation, the nucleon is treated as free, and the on-shell relation fixes one component of the nucleon momentum: $p^{2}=M_{N}^{2}$. However, one has to go beyond PWIA in order to describe (semi-)exclusive observables, such as the nucleon knock-out reactions. In particular, one has to take into account the nucleon off-shellness due to the initial state interaction (ISI).

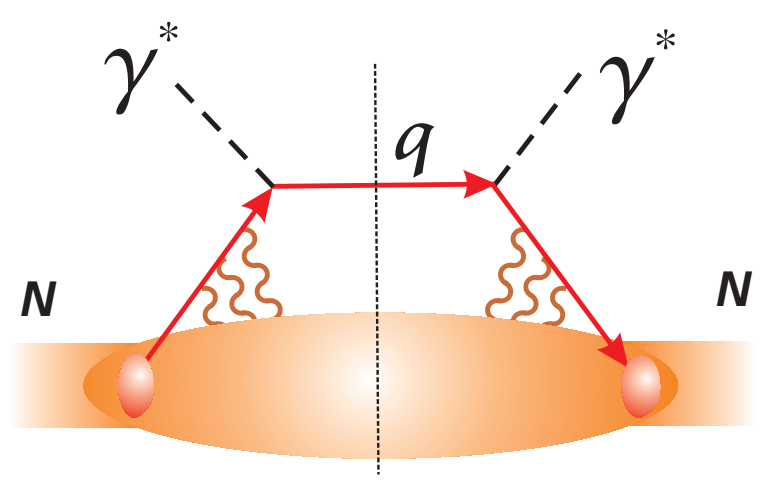

Figure 1: The handbag graph for DIS and the relevant initial state interactions that could build up a finite parton width

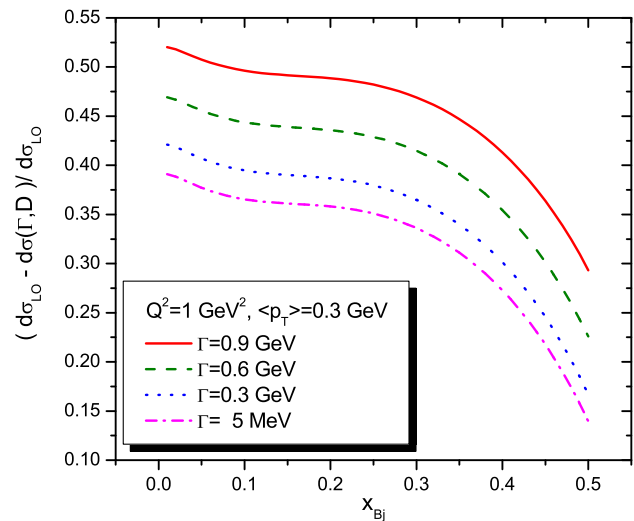

Figure 2: Deviation of the calculated DIS cross section from parton model for a range of parton spectral function widths. 


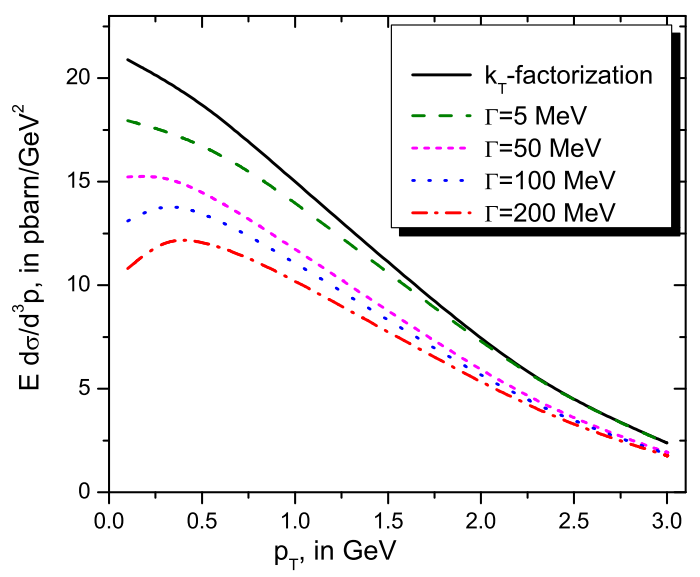

Figure 3: Triple differential Drell-Yan cross section in our model for different values of the parton width $\Gamma$. The solid line is at $\Gamma=0$.

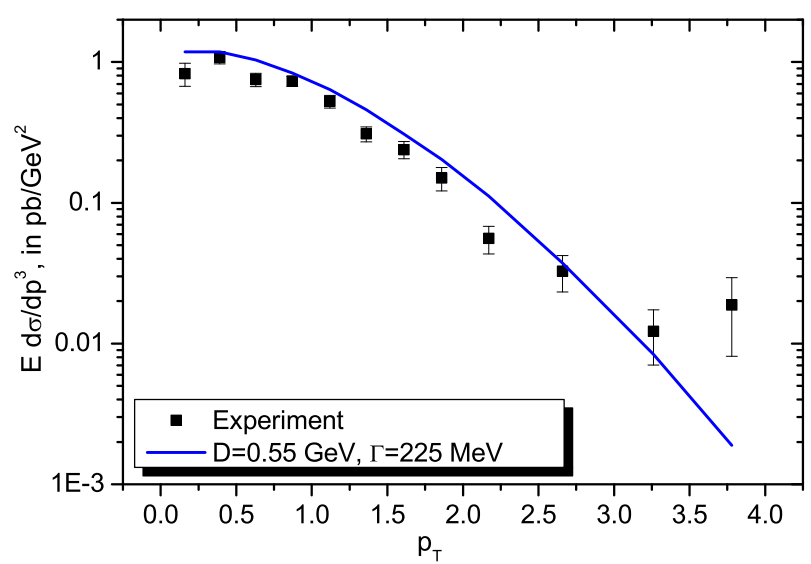

Figure 4: Calculated Drell-Yan cross section compared to the data of the Fermilab experiment 866 for $p p \rightarrow \mu^{+} \mu^{-}+X$ at $800 \mathrm{GeV}$ incident $p$ energy.

In case of fully inclusive $e p$ DIS, the factorization theorem justifies the use of the handbag diagram at high momentum transfer squared $Q^{2}$. In the limit $Q^{2} \rightarrow \infty$, the quark can be considered free and its momentum satisfies the on-shell relation $p^{-}=p^{0}-p^{z}=0$. On the other hand, a quark bound in nucleon is always off-shell, i.e. $p^{-}$is not fixed to 0 , but is distributed with some finite width. At moderate $Q^{2}$ the effect of quark off-shellness on the observable cross section might be considerable. The handbag diagram for the DIS with the relevant initial state interactions is presented in Fig. 1. Following the suggestion of [1], we calculated the quark off-shellness effects by dressing the incoming quark lines with model spectral functions. We use the factorization assumption to write the DIS cross section as follows:

$$
d \sigma_{\mathrm{DIS}}=\sum_{i} g_{i}\left(\xi, \vec{p}_{\perp}, p^{-}\right) \otimes d \hat{\sigma}_{i}\left(\xi, \vec{p}_{\perp}, p^{-}\right),
$$

where $d \hat{\sigma}_{i}$ is the cross section of the sub-process $e q_{i} \rightarrow e q_{i}, \xi=p^{+} / P_{N}^{+}$is the fraction of nucleon light cone momentum carried by the struck quark, $p^{-}$is the minus component of the quark's momentum and $\vec{p}_{\perp}$ is its transverse momentum. $d \hat{\sigma}_{i}$ is convoluted with a function $g_{i}\left(\xi, \vec{p}_{\perp}, p^{-}\right)$giving the probability to find a quark of flavour $i$ with momentum $p$ and virtuality (off-shellness) $m^{2}$ in the nucleon $\left(m^{2} \equiv p^{+} p^{-}-\vec{p}_{\perp}^{2}\right)$. Formula (11) is easily generalized for the case of two partons in the initial state to describe the Drell-Yan process. In addition, we propose the following ansatz:

$$
g_{i}\left(\xi, \vec{p}_{\perp}, p^{-}\right)=f_{i}\left(\xi, \vec{p}_{\perp}\right) A(m, \Gamma),
$$

where $f_{i}\left(\xi, \vec{p}_{\perp}\right)$ are unintegrated parton distributions [2], $A(m, \Gamma)$ quark spectral function, for which we use the Breit-Wigner parametrization with constant width.

The results of our calculations for fully inclusive DIS are presented in Figure 2, The calculated Drell-Yan cross section for different values of $\Gamma$ are given in Figure 3, From the comparison to the data if the Fermilab experiment E866 [3], we estimated the quark width in proton to be of the order of 200 $\mathrm{MeV}$. The example of our description of the data is presented in Figure 4 in one bin of muon pair mass $7.2 \leq M \leq 8.7 \mathrm{GeV}$ and Feynman $\mathrm{x}-0.05 \leq x_{F} \leq 0.15$. The data can be described without a K-factor.

\section{References}

[1] O. Benhar, preprint, nucl-th/0204042.

[2] A. A. Henneman, D. Boer and P. J. Mulders, Nucl. Phys. B 620 (2002) 331, hep-ph/0104271.

[3] J. C. Webb, New Mexico State University, PhD thesis, hep-ex/0301031. 